\title{
LINEAR OPERATOR EQUATIONS
}

\section{GUNTER LUMER AND MARVIN ROSENBLUM ${ }^{1}$}

I. Introduction. Let $a$ be a complex Banach algebra with identity $e$, and let $B$ be the Banach algebra of operators on $a$ considered as a Banach space.

Given $u, v \in Q$, let $T \in B$ be defined by $T x=u x-x v$. This operator was studied in [4] where it was shown that if $\sigma(x, a)$ denotes the spectrum of $x \in Q$, then

$$
\sigma(T, B) \subset \sigma(u, a)-\sigma(v, a)
$$

and

$$
f(T) x=\frac{1}{2 \pi i} \int_{c} f(u-\lambda e) x(\lambda e-v)^{-1} d \lambda,
$$

where $f$ is holomorphic on $\sigma(u, a)-\sigma(v, Q)$, and $c$ is a suitable contour.

In the present note we study the operator $S \in B$ defined by $S x$ $=\sum_{j=1}^{N} u_{j} x v_{j},\left\{u_{j}\right\}$ and $\left\{v_{j}\right\}$ being commutative subsets of $a ;$ but it is understood that $\left\{u_{j}\right\}$ need not commute with $\left\{v_{j}\right\}$. In a generic way, we shall refer to such an operator as an "elementary operator." ${ }^{2}$ Of course this is related to the problem of solving in $a$ a general system of linear equations. We generalize the analysis made in [4] covering the $u x-x v$ case, and extend the following unpublished theorem of D. C. Kleinecke:

(1.3) If $Q$ is the Banach algebra of all operators on a Banach space, then " $C$ " may be replaced by " $=$ " in (1.1).

II. Mappings. A simplification of the theory developed in [4] is achieved by introducing the mappings ${ }^{+}$and - from $a$ into $B$, that associates with any $u \in Q$, the operators $u^{+} \in B$ defined by $u^{+}(x)$

Presented to the Society April 26, 1958; received by the editors February 20, 1958.

1 This article was written while the first named of the authors was a Fellow of the John S. Guggenheim Foundation. The work of M. Rosenblum was partially supported by the United States Air Force through the Air Force Office of Scientific Research of the Air Research and Development Command, under contract No. AF $49(638)-72$ at the University of Virginia.

2 Because of the type of linear equations in which we are interested, we consider each elementary operator to be generated by a finite collection of $\{u\}$ and $\{v\}$. The theory could, however, be extended farther. For example, essentially the same results would hold for $S x=\sum_{1}^{\infty} u_{j} x v_{j}$ if $\sum_{1}^{\infty}\left\|u_{j}\right\|\left\|v_{j}\right\|<+\infty$. 
$=u x$, and $u^{-} \in B$, defined by $u^{-}(x)=x u$. Now, first of all let us point out the simple and yet important fact that for any $u, v \in a, u^{+} v^{-}$ $=v^{-} u^{+}$as direct verification shows.

Furthermore, put $a^{+}=\left\{u^{+}: u \in \mathcal{u}\right\} ; \mathbb{Q}^{-}=\left\{u^{-}: u \in Q\right\}$.

Lemma $1 .+i$ is an isometric isomorphic mapping of the Banach algebra $a$ onto the Banach algebra $a^{+} .{ }^{-}$is an isometric anti-isomorphism of $a$ onto $Q^{-}$.

Proof. Direct verification.

LEMMA 2.

$$
\begin{aligned}
& \sigma(u, \mathbb{Q})=\sigma\left(u^{+}, \mathbb{B}\right), \\
& \sigma(u, \mathbb{Q})=\sigma\left(u^{-}, \mathbb{B}\right) .
\end{aligned}
$$

Proof. Let us prove (2.1). Lemma 1 implies that $\sigma(u, a)$ $=\sigma\left(u^{+}, Q^{+}\right) \supset \sigma\left(u^{+}, \otimes\right)$. Therefore it suffices to show that $0 \notin \sigma\left(u^{+}, B\right)$ implies $0 \notin \sigma(u, Q)$. Suppose $0 \notin \sigma\left(u^{+}, \bigotimes\right)$, then there exists $W \in B$ such that $u^{+} W=W u^{+}=e^{+}$. Hence, $u W(e)=W(u)=e$. So $u(e-W(e) u)$ $=u-u W(u)=0$ and $u^{+}(e-W(e) u)=0$. Since $u^{+}$is regular, $e-W(e) u$ $=0$, and $u W(e)=W(e) u=e$, so $0 \notin \sigma(u, \mathfrak{A})$.

It seems pertinent to make the following remark: $f(\quad)$ being a complex valued function, holomorphic in some domain $D$, its Banachalgebraic extension in $Q$ is defined in the usual way, at any point $a \in Q$ such that $\sigma(a, a) \subset D$, by

$$
f(a)=\frac{1}{2 \pi i} \int_{c} f(\lambda) R(\lambda, a) d \lambda
$$

where $c$ is a suitable contour, and $R(\lambda, a)=(\lambda e-a)^{-1}$. It thus follows from Lemma 2, that the extension of $f$ in $a$ is defined at $a \in a$ if, and only if, the extension of $f$ in $B$ is defined at $a^{+}$and $a^{-}$. Furthermore, as an immediate consequence of Lemma 1, we have the following remark, which for convenience of reference we state as

LemMa 3. $f$ being a complex valued holomorphic function whose extension in $a$ is defined at $a \in a$, we have

$$
\begin{aligned}
& (f(a))^{+}=f\left(a^{+}\right), \\
& (f(a))^{-}=f\left(a^{-}\right) .
\end{aligned}
$$

Incidentally from this the well known relation $e^{T}(x)=e^{u} x e^{-v}$ follows immediately, since $e^{T}(x)=e^{u^{+}-v^{-}}(x)=e^{u^{+}} e^{-v^{-}}(x)=\left(e^{u}\right)^{+}\left(e^{-v}\right)^{-} x$ $=e^{u} x e^{-v}$. 
III. The spectrum of $S$. Here we generalize (1.1).

Theorem 4.

$$
\sigma(S, Q) \subset \sum_{j=1}^{N} \sigma\left(u_{j}, Q\right) \sigma\left(v_{j}, a\right) .
$$

Proof. Let $B_{0}$ be the subalgebra generated by $u_{1}^{+}, \cdots, u_{N}^{+}$, $v_{\overline{1}}^{-}, \cdots, v_{N}^{-}, e^{+}$. Since any two elements of $B_{0}$ commute, it is easy to show (see for instance [3, Lemma 1]) that there exists a commutative Banach algebra $B_{1}$, such that $B_{0} \subset B_{1} \subset B$ and $\sigma\left(w, B_{1}\right)$ $=\sigma(w, B)$ for any $w$ in $B_{0}$.

Let $\mu$ be a multiplicative linear functional on $\Theta_{1}$ (see $[1$, p. 127]). Since $S=\sum_{j=1}^{N} u_{j}^{+} v_{j}^{-} \in \bigotimes_{1}, \mu(S)=\sum_{j=1}^{N} \mu\left(u_{j}^{+}\right) \mu\left(v_{j}^{-}\right)$. But the ranges of values of $\mu\left(u_{j}^{+}\right), \mu\left(v_{j}^{-}\right), \mu(S)$ as $\mu$ ranges over all multiplicative linear functionals on $\Theta_{1}$ are $\sigma\left(u_{j}^{+}, \Theta_{1}\right), \sigma\left(v_{j}^{-}, \Theta_{1}\right), \sigma\left(S, \Theta_{1}\right)$ respectively. Thus

$$
\sigma(S, \bigotimes) \subset \sum_{j=1}^{N} \sigma\left(u_{j}^{+}, \bigotimes_{1}\right) \sigma\left(v_{j}^{-}, \bigotimes_{1}\right),
$$

so

$$
\sigma(S, B) \subset \sum_{j=1}^{N} \sigma\left(u_{j}^{+}, B\right) \sigma\left(v_{j}^{-}, B\right) .
$$

By Lemma 2 this implies Theorem 2.

If the $u_{j}$ as well as the $v_{j}$ are functionally related we can prove a stronger result.

Theorem 5. Suppose $u, v \in a$ and that $f_{j}(\lambda), g_{j}(\lambda), j=1,2, \cdots, N$ are holomorphic in domains containing $\sigma(u), \sigma(v)$ respectively. Define $R \in B$ by

$$
R x=\sum_{j=1}^{N} f_{j}(u) x g_{j}(v), \quad x \in Q .
$$

Then $\sigma(R) \subset\left\{\sum_{j=1}^{N} f_{j}(\alpha) g_{j}(\beta): \alpha \in \sigma(u)\right.$ and $\left.\beta \in \sigma(v)\right\}$.

Proof. We proceed as in Theorem 2. Here $B_{0}$ is the subalgebra generated by $\left\{u^{+}, v^{-}, e^{+}\right\}$, and $\mu(R)=\sum_{j=1}^{N} \mu\left(f_{j}\left(u^{+}\right)\right) \mu\left(g_{j}\left(v^{-}\right)\right)$. By $[1$, p. 127],

$$
\mu(R)=\sum_{j=1}^{N} f_{j}\left(\mu\left(u^{+}\right)\right) g_{j}\left(\mu\left(v^{-}\right)\right) .
$$

Let $u$ range over all multiplicative linear functionals on $B_{1}$. Then 


$$
\sigma\left(R, \bigotimes_{1}\right) \subset\left\{\sum_{j=1}^{N} f_{j}(\alpha) g_{j}(\beta): \alpha \in \sigma\left(u^{+}, \bigotimes_{1}\right) \text { and } \beta \in \sigma\left(v^{-}, \bigotimes_{1}\right)\right\} .
$$

Finally, $\sigma\left(u^{+}, B_{1}\right)=\sigma\left(u^{+}, B\right)=\sigma(u, Q)$ and similarly $\sigma\left(v^{-}, B\right)=\sigma(v, Q)$.

It is quite easily seen that equality, instead of inclusion in the relation (3.1) of Theorem 4 need not hold in general. And yet, since the representation of an elementary operator in terms of $a^{+}$and $a^{-}$is generally not unique it is natural to ask what happens to the right hand of relation (3.1), when we consider all possible representations of an elementary operator.

It turns out that in general, we cannot say anything more than what Theorem 4 already tells us.

Let us simply examine the case $K=u^{+}+v^{-}$. It is trivial that $u^{+}+v^{-}=w^{+}+z^{-}$if and only if $w=u-c$ and $z=v+c$ with $c \in \mathcal{C}(Q)$; where $\mathfrak{C}(\boldsymbol{Q})$ denotes the center of $\boldsymbol{Q}$.

Consider more in particular the following situation: Let $\mathfrak{H}$ be a Hilbert space, $\mathfrak{J}$ a nontrivial subspace, $P$ the projection on $\mathfrak{J}$, and $Q$ the projection on $J^{\perp}$. Let $a$ be the Banach algebra of operators on $\mathfrak{F}$, and put $a_{1}=\{W \in a: W P=P W\} ; \quad a_{2}=\{V \in a: V P=P V P\}$. Let us denote by $B_{1}$ and $B_{2}$, the algebras of operators on $Q_{1}, Q_{2}$. $Q_{1}$ and $a_{2}$ are Banach algebras with unit I. Consider the identity operator on $a_{1}$, which can be written either $P^{+}+Q^{-}$or $I^{+}$; and $\sigma\left(P, a_{1}\right)$ $+\sigma\left(Q, Q_{1}\right)=\{0,1,2\} \neq\{1\}=\sigma\left(I^{+}, Q_{1}\right)$. In this case different sets for the right hand side in relation (3.1) arise for different representations of the identity operator; but the intersection of these sets equals the left hand side of the relation. This, however, is not a general situation either. In fact, consider $a_{2}$, and the corresponding elementary operator defined by $P^{+}+Q^{-}=K$. It can be shown that $0 \notin \sigma\left(K, Q_{2}\right) \neq \sigma\left(P, Q_{2}\right)+\sigma\left(Q, Q_{2}\right)$. On the other hand, it is easy to verify that the center of $a_{2}$ contains only scalar multiples of the identity and thus no different sets arise from different representations of $K$.

We now turn our attention to the operational calculus for elementary operators.

IV. Cauchy formulas. We shall first derive a representation formula for certain holomorphic functions of several commuting Banach algebra elements and then apply that result to generalize (1.2).

THEOREM 6. Let $u, v, w$ be commuting elements of a and let $P\left(\lambda_{1}, \lambda_{2}, \lambda_{3}\right)$ be a polynomial in the complex variables $\lambda_{1}, \lambda_{2}, \lambda_{3}$. Suppose $f(\lambda)$ is holomorphic in a domain that contains $P(\sigma(u), \sigma(v), \sigma(w))$. Then there exists a Cauchy domain $D_{1} \supset \sigma(u)$, (see [6]), such that 


$$
f(P(u, v, w))=\frac{1}{2 \pi i} \int_{\partial D_{1}} f(P(\lambda, v, w))(\lambda e-u)^{-1} d \lambda
$$

where $\partial D_{1}$ is the boundary of $D_{1}$.

Proof. Since $\sigma(u+v) \subset \sigma(u)+\sigma(v)$ and $\sigma(u \cdot v) \subset \sigma(u) \cdot \sigma(v)$ it follows that $\sigma(P(u, v, w)) \subset P(\sigma(u), \sigma(v), \sigma(w))$. Let

$$
D_{0} \supset P(\sigma(u), \sigma(v), \sigma(w))
$$

be a Cauchy domain such that $\bar{D}_{0}$ is contained in the domain of regularity of $f(\lambda)$. Then, by definition

$$
f(P(u, v, w))=\frac{1}{2 \pi i} \int_{\partial D_{0}} f(\xi)(\xi e-P(u, v, w))^{-1} d \xi .
$$

Choose the Cauchy domain $D_{1} \supset \sigma(u)$ so that

$$
D_{0} \supset P\left(\bar{D}_{1}, \sigma(v), \sigma(w)\right) \text {. }
$$

Suppose that $\xi \in \partial D_{0}$ and $\lambda \in \partial D_{1}$. Then $P(\lambda, v, w)-P(u, v, w)$ $=(\lambda e-u) Q$, and the partial fraction expansion

$$
\begin{aligned}
{\left[(\xi e-P(\lambda, v, w))^{-1} Q+(\lambda e-u)^{-1}\right][\xi e-P(u, v, w)]^{-1} } \\
=[\xi e-P(\lambda, v, w)]^{-1}[\lambda e-u]^{-1}
\end{aligned}
$$

is valid. If $\xi \in \partial D_{0}$, then

$(* * *)$

$$
\begin{aligned}
\frac{1}{2 \pi i} \int_{\partial D_{1}}\left[(\xi e-P(\lambda, v, w))^{-1} Q+(\lambda e-u)^{-1}\right] d \lambda & \\
& =\frac{1}{2 \pi i} \int_{\partial D_{1}}(\lambda e-u)^{-1} d \lambda=e .
\end{aligned}
$$

By use of $(*),(* *),(* * *)$ we see that

$$
\begin{aligned}
f(P(u, v, w))= & \frac{1}{(2 \pi i)^{2}} \int_{\partial D_{0}} \int_{\partial D_{1}} f(\xi)\left[(\xi e-P(\lambda, v, w))^{-1} Q\right. \\
& \left.+(\lambda e-u)^{-1}\right][\xi e-P(u, v, w)]^{-1} d \xi d \lambda \\
= & \frac{1}{2 \pi i} \int_{\partial D_{1}} f\left(P(\lambda, v, w)(\lambda e-u)^{-1} d \lambda .\right.
\end{aligned}
$$

THEOREM 7. Let $u_{1}, \cdots, u_{N}, v_{1}, \cdots, v_{N}$ be commuting elements of a and suppose $f(\lambda)$ is holomorphic in a domain that contains $\sum_{j=1}^{N} \sigma\left(u_{j}\right) \sigma\left(v_{j}\right)$. Then there exist Cauchy domains $D_{1} \supset \sigma\left(v_{1}\right), \cdots$, $D_{N} \supset \sigma\left(v_{N}\right)$ such that 


$$
f\left(\sum_{j=1}^{N} u_{j} v_{j}\right)=\frac{1}{(2 \pi i)^{N}} \int_{\partial D_{1}} \cdots \int_{\partial D_{N}} f\left(\sum_{j=1}^{N} \lambda_{j} u_{j}\right) \prod_{j=1}^{N}\left(\lambda_{j} e-v_{j}\right)^{-1} d \lambda_{j} .
$$

Proof. Iterated use of Theorem 6. For a similar result see [5].

We now generalize (1.2).

THEOREM 8. Let $f(\lambda)$ be holomorphic in a domain that contains $\sum_{j=1}^{N} \sigma\left(u_{j}\right) \sigma\left(v_{j}\right)$. Then there exist Cauchy domains $D_{1} \supset \sigma\left(u_{1}\right), \cdots$, $D_{N} \supset \sigma\left(u_{N}\right)$ such that for any $x \in Q$,

$$
f(S)(x)=\frac{1}{(2 \pi i)^{N}} \int_{\partial D_{1}} \cdots \int_{\partial D_{N}} f\left(\sum_{j=1}^{N} \lambda_{j} u_{j}\right) x \prod_{j=1}^{N}\left(\lambda_{j} e-v_{j}\right)^{-1} d \lambda_{j} .
$$

Proof. $u_{1}^{+}, \cdots, u_{N}^{+}, v_{1}^{-}, \cdots, v_{\bar{N}}$ are commuting elements of $B$ and by Lemma $2 f(\lambda)$ is holomorphic in a domain that contains $\sum_{j=1}^{N} \sigma\left(u_{j}^{+}\right) \sigma\left(v_{j}^{-}\right)$. Hence we know from Theorem 7 that

$$
f(S)(x)=\frac{1}{(2 \pi i)^{N}} \int_{\partial D_{1}} \cdots \int_{\partial D_{N}}\left[f\left(\sum_{j=1}^{N} \lambda_{j} u_{j}^{+}\right) \prod_{j=1}^{N}\left(\lambda_{j} e^{-}-v_{j}^{-}\right)^{-1} d \lambda_{j}\right] x .
$$

We now use Lemma 3. $\left(\lambda_{j} e^{-}-v_{j}^{-}\right)^{-1}=\left[\left(\lambda_{j} e-v_{j}\right)^{-}\right]^{-1}=\left[\left(\lambda_{j} e-v_{j}\right)^{-1}\right]^{-}$; and

Thus

$$
f\left(\sum_{j=1}^{N} \lambda_{j} u_{j}^{+}\right)=\left[f\left(\sum_{j=1}^{N} \lambda_{j} u_{j}\right)\right]^{+} .
$$

$$
f\left(\sum_{j=1}^{N} \lambda_{j} u_{j}^{+}\right) \prod_{j=1}^{N}\left(\lambda_{j} e^{-}-v_{j}^{-}\right) x=f\left(\sum_{j=1}^{N} \lambda_{j} u_{j}\right) x \prod_{j=1}^{N}\left(\lambda_{j} e-v_{j}\right)^{-1},
$$

and the proof is complete.

V. Kleinecke's Theorem. It is of interest to study generalizations of (1.3). If $S \in Q$ is defined by $S x=u_{1} x+u_{2} x, x \in Q$, then by Lemma 2, $\sigma(S)=\sigma\left(u_{1}+u_{2}\right)$, which need not equal $\sigma\left(u_{1}\right)+\sigma\left(u_{2}\right)$ even though $u_{1}, u_{2}$ commute. We shall therefore confine our attention to $R \in \Theta$, as defined in Theorem 5 .

Suppose $\lambda \in \sigma(R)$. By Theorem 5 we know that there exists $\alpha \in \sigma(u)$ and $\beta \in \sigma(v)$ such that $\lambda=\sum_{j=1}^{N} f_{j}(\alpha) g_{j}(\beta)$. In Theorem 9 we list criteria that will allow us to infer from $\alpha \in \sigma(u)$ and $\beta \in \sigma(v)$ that $\lambda=\sum_{j=1}^{N} f_{j}(\alpha) g_{j}(\beta) \in \sigma(R)$.

Suppose $x \in Q$, and $\left\{x_{n}\right\}$ is a sequence of vectors in $Q$; we shall say that $\left\{x_{n}\right\}$ left (right) zero-divides $x$ if $\left\|x_{n}\right\|=1, n=0,1, \cdots$, and $x_{n} x \rightarrow 0\left(x x_{n} \rightarrow 0\right)$.

Theorem 9. Suppose $\left\{z_{n}\right\}$, is a sequence of unit vectors in $a$ and (i) $x_{n}$ left or right zero-divides $u-\alpha e$; 
(ii) $y_{n}$ left or right zero-divides $v-\beta e$; and

(iii) $x_{n} z_{n} y_{n}$ does not converge to 0 .

Then $\lambda \in \sigma(R)$.

Proof. Since $\left\{x_{n}\right\}$ left (right) zero-divides $u-\alpha e,\left\{x_{n}\right\}$ left (right) zero-divides $f_{j}(u)-f_{j}(\alpha) e, j=1,2, \cdots, N$. (See [1, p. 123]). A similar statement holds for $\left\{y_{n}\right\}$ and $g_{j}(u)-g_{j}(\beta) e$.

The proof of Theorem 9 is indirect. We therefore make the assumption (*) $\lambda \bigoplus \sigma(R)$, and consider four cases:

CASE 1. Suppose

(i) $\left\{x_{n}\right\}$ right zero-divides $u-\alpha e$, and

(ii) $\left\{y_{n}\right\}$ right zero-divides $v-\beta e$.

By $(*)$ there exists a uniformly bounded sequence $\left\{p_{n}\right\}$ such that $\left(R-\lambda e^{+}\right) p_{n}=x_{n} z_{n}$. Since

$$
\begin{gathered}
\left(f_{j}(u)-f_{j}(\alpha) e\right) x_{n} \rightarrow 0, \\
\left(R-\lambda e^{+}\right)\left[\left(f_{j}(u)-f_{j}(\alpha) e\right) p_{n}\right]=\left(f_{j}(u)-f_{j}(\alpha) e\right) x_{n} z_{n} \rightarrow 0,
\end{gathered}
$$

and by $(*)$ this guarantees that $\left(f_{j}(u)-f_{j}(\alpha) e\right) p_{n} \rightarrow 0$. But then

$$
\begin{aligned}
x_{n} z_{n} y_{n} & =\left[\left(R-\lambda e^{+}\right) p_{n}\right] y_{n}=\sum_{j=1}^{N}\left[f_{j}(u) p_{n} g_{j}(v) y_{n}-f_{j}(\alpha) g_{j}(\beta) p_{n} y_{n}\right] \\
& =\sum_{j=1}^{N}\left[\left(f_{j}(u)-f_{j}(\alpha) e\right) p_{n} g_{j}(v) y_{n}+f_{j}(\alpha) p_{n}\left(g_{j}(v)-g_{j}(\beta) e\right) y_{n}\right] .
\end{aligned}
$$

But each of these terms goes to 0 as $n \rightarrow \infty$, contradicting (iii). Thus $\lambda \in \sigma(R)$.

CASE 2. Suppose

(i) $\left\{x_{n}\right\}$ left zero-divides $u-\alpha e$ and

(ii) $\left\{y_{n}\right\}$ left zero-divides $v-\beta e$.

By $(*)$ there exists a uniformly bounded sequence $\left\{p_{n}\right\}$ such that $\left(R-\lambda e^{+}\right) p_{n}=z_{n} y_{n} .\left(R-\lambda e^{+}\right)\left[p_{n}\left(g_{j}(v)-g_{j}(\beta) e\right)\right]=z_{n} y_{n}\left(g_{j}(v)-g_{j}(\beta) e\right)$ $\rightarrow 0$, and by the continuity of $\left(R-\lambda e^{+}\right)^{-1}, p_{n}\left(g_{j}(v)-g_{j}(\beta) e\right) \rightarrow 0$. Now

$$
\begin{aligned}
x_{n} z_{n} y_{n} & =x_{n}\left[\left(R-\lambda e^{+}\right) p\right] \\
& =\sum_{j=1}^{N}\left[x_{n}\left(f_{j}(u)-f_{j}(\alpha) e\right) p_{n} g_{j}(v)+f_{j}\left(\lambda_{j}\right) x_{n} p_{n}\left(g_{j}(v)-g_{j}(\beta) e\right)\right] \rightarrow 0,
\end{aligned}
$$

contradicting (iii). Thus $\lambda \in \sigma(R)$.

Case 3. Suppose

(i) $\left\{x_{n}\right\}$ left zero-divides $u-\alpha e$ and

(ii) $\left\{y_{n}\right\}$ right zero-divides $v-\beta e$.

There exists a uniformly bounded sequence $\left\{p_{n}\right\}$ such that 
$\left(R-\lambda e^{+}\right) p_{n}=z_{n}$. Hence $x_{n} z_{n} y_{n}=\sum_{j=1}^{N} x_{n} f_{j}(u) p_{n}\left(g_{j}(v)-g_{j}(\beta) e\right) y_{n}$ $+\sum_{j=1}^{N} g_{j}(\beta) x_{n}\left(f_{j}(u)-f_{j}(\alpha) e\right) p_{n} y_{n} \rightarrow 0$, contradicting $(*)$.

CASE 4. Suppose

(i) $\left\{x_{n}\right\}$ right zero-divides $u-\alpha e$, and

(ii) $\left\{y_{n}\right\}$ left zero-divides $v-\beta e$.

Then

$$
\begin{aligned}
\left(R-\lambda e^{+}\right)\left(x_{n} z_{n} y_{n}\right)= & \sum_{j=1}^{N}\left(f_{j}(u)-f_{j}(\alpha) e\right) x_{n} z_{n} y_{n} g_{j}(v) \\
& +\sum_{j=1}^{N} f_{j}(\alpha) x_{n} z_{n} y_{n}\left[g_{j}(v)-g_{j}(\beta) e\right] \rightarrow 0 .
\end{aligned}
$$

So again $\lambda \in \sigma(R)$.

Next we generalize Kleinecke's theorem.

Theorem 10. Suppose $a$ is the Banach algebra of endomorphisms on a Banach space e. Then

$$
\sigma(R)=\left\{\sum_{j=1}^{N} f_{j}(\alpha) g_{j}(\beta): \alpha \in \sigma(u) \text { and } \beta \in \sigma(v)\right\} .
$$

Proof. Suppose $\alpha \in \sigma(u), \beta \in \sigma(v)$. Since $u, v$, are operators on a Banach space, it follows that there exist sequences $x_{n}, y_{n}$ that zerodivide $u-\alpha e$ and $v-\beta e$ respectively. Choose $\left\{\gamma_{n}\right\} \subset \mathbb{e}$ and $\left\{\delta_{n}^{*}\right\} \subset \mathbb{C}^{*}$ such that $\left\|x_{n}\left(\gamma_{n}\right)\right\|=1$ and $\left\|y_{n}^{*}\left(\delta_{n}^{*}\right)\right\|=1$. Put $\left\langle\cdot, \delta_{n}^{*}\right\rangle \gamma_{n}=z_{n} \in Q$, and note that $x_{n} z_{n} y_{n}=\left\langle\cdot, y_{n}^{*} \delta_{n}^{*}\right\rangle x_{n}\left(\gamma_{n}\right)$ does not converge to 0 . Hence by Theorem $9, \lambda \in \sigma(R)$, and by Theorem 5 the proof is complete.

VI. Linear equation. We shall relate our theory to some results concerning the general linear matrix equation $\sum_{j=1}^{N} u_{j} x v_{j}=w$, where $u_{1}, \cdots, u_{N}, v_{1}, \cdots, v_{N}, w$ are elements in a complex full matrix algebra $a$. The operator $T=\sum_{j=1}^{N} u_{j}^{+} \cdot v_{j}^{-}$can be written $T$ $=\sum_{j=1}^{N} u_{j} \otimes v_{j}$, where $u_{j} \otimes v_{j}$ is the direct product of $u_{j}$ and $v_{j}$. If $u_{j}$ and $v_{j}$ separately commute and $0 \notin \sum_{j=1}^{N} \sigma\left(u_{j}\right) \sigma\left(v_{j}\right)$, then Theorem 8 gives us an integral representation for $T^{-1}$. Theorem 10 is a generalization of the following theorem of Stephanos [2, p. 83]:

The characteristic roots of $\sum_{i, j=1}^{N} c_{i j} u^{i} \otimes v^{j}$ are the numbers $\sum_{i, j} c_{i j} \alpha_{p}^{q} \beta_{q}^{j}$, where $\left\{\alpha_{p}\right\},\left\{\beta_{q}\right\}$ are the characteristic roots of $u$ and $v$ respectively.

Finally, consider the result of Sylvester $[2$, p. 90].

The matrix equation $u x=x v, u, v \in Q$ has a non-null solution $x \in Q$ if and only if $u$ and $v$ have a common characteristic root.

This is an immediate consequence of Theorem 10, which shows that if $T x=u x-x v$, then $\sigma(u)-\sigma(v)=\sigma(T)$. 
VII. Systems of linear equations. We will only consider here the explicit system of $n$ equations with $n$ unknowns whose general form is now

$$
\sum_{j} \sum_{k} u_{i j}(k) x_{j} v_{i j}(k)=y_{i} \quad(i=1, \cdots, n) .
$$

In absence of commutativity the usual theory of linear equations breaks down. However, if we make a partial commutativity assumption, that is if we assume as before that all $\{u\}$ commute, and all $\{v\}$ commute, but separately, the system (7.1) can be written in the form

$$
\sum_{j} S_{i j} x_{j}=y_{i} \quad(i=1, \cdots, n)
$$

where the $S_{i j}$ are elementary operators. We now generalize the sufficient condition for the existence of a solution of (7.2) in terms of the coefficients in $\alpha$. Denote by det $\left[\alpha_{i j}\right]$ the $n \times n$ determinant whose generic entry is $\alpha_{i j}$. If $\alpha_{i j}$ are sets of complex numbers we put det $\left[\alpha_{i j}\right]$ $=\left\{\operatorname{det}\left[\lambda_{i j}\right]: \lambda_{i j} \in \alpha_{i j}\right\}$.

THEOREM 11. Under the assumption that $\{u\}$ and $\{v\}$ commute separately, a sufficient condition for the system (7.1) to have a solution linear and continuous in $\left(y_{1}, y_{2}, \cdots, y_{n}\right)$ is that

$$
0 \notin \operatorname{det}\left[\sum_{k} \sigma\left(u_{i j}(k), \mathfrak{a}\right) \sigma\left(v_{i j}(k), \mathfrak{Q}\right)\right] .
$$

Proof. Consider the system of linear equations in $B$ :

$$
\sum_{j} S_{i j} x_{j}=y_{i}^{+} \quad(i=1, \cdots, n) .
$$

Here the $S_{i j}$ act as simple coefficients, and form a commutative subset of $B$. The general theory of matrices and determinants is applicable here provided the $y_{j}^{+}$are always kept to the right of the $S_{j k}$. Thus (7.3) has a solution (linear and continuous in the $y_{j}^{+}$) if det $S_{i j}$ is invertible as an element of $B$. Suppose this is so, then we obtain a solution of the original equations (7.1) (which is again linear and continuous) by applying $e \in Q$ to both sides of the system (7.3) and setting $x_{i}=X_{i}(e)$. It thus remains only to show that $0 \notin \sigma\left(\operatorname{det}\left[S_{i j}\right], ß\right)$. But the same multiplicative functionals argument of Theorem 4 shows that

$$
\sigma\left(\operatorname{det}\left[S_{i j}\right], ß\right) \subset \operatorname{det}\left[\sigma\left(S_{i j}, \bigotimes\right)\right]
$$

and from 3.1 


$$
\sigma\left(\operatorname{det}\left[S_{i j}\right], \beta\right) \subset \operatorname{det}\left[\sum_{k} \sigma\left(u_{i j}(k), a\right) \sigma\left(v_{i j}(k), a\right)\right] .
$$

Thus $0 \in \sigma\left(\operatorname{det}\left[S_{i j}\right], ß\right)$ would contradict the hypothesis.

\section{REFERENCES}

1. Einar Hille, Functional analysis and semigroups, Amer. Math. Soc. Colloquium Publication, vol. 31, 1948.

2. C. C. MacDuffee, The theory of matrices, Ergebnisse der Math., 1933.

3. Günter Lumer, Conmutadores en algebras de Banach, Publ. Instituto de Matematica, Montevideo vol. 3 (1957) pp. 53-55.

4. Marvin Rosenblum, On the operator equation $B X-X A=Q$, Duke Math. J. vol. 23 (1956) pp. 263-269.

5. M. A. Rutman, On certain operator equations in a partially ordered space which have applications to the theory of stability according to Lyapunov, Dokl. Akad. Nauk SSSR N.S. vol. 101 (1955) pp. 217-220.

6. A. E. Taylor, Spectral theory of closed distributive operators, Acta Mathematica vol. 84 (1951) pp. 189-224.

University OF Chicago AND

UNIVERSITY OF VIRGINIA 